\title{
Allergens in Japanese patients with allergic conjunctivitis in autumn
}

\begin{abstract}
Purpose The purpose of the current study is to evaluate the relation between various specific class E immunoglobulins (IgE) in the serum and allergic conjunctivitis in autumn. Methods Total IgE and specific IgE to 12 inhalant allergens were measured using the CAP system in 32 patients with allergic conjunctivitis in spring (spring group), 27 patients with allergic conjunctivitis in autumn (autumn group), and 40 healthy volunteers (control group).

Results Specific IgE levels caused by house dust, Dermatophagoides pteronyssinus, and orchard grass were higher in the autumn group than in the spring group. The highest positivity rate for a specific allergen was $51.9 \%$ for house dust, followed by $D$. pteronyssinus $\mathbf{( 4 8 . 1 \% )}$ in the autumn group, while the highest rate was $68.8 \%$ for cedar pollen, followed by cypress pollen $(59.4 \%)$ in the spring group. Correlation analysis showed that house dust was significantly correlated with animal epithelia, D. pteronyssinus, acarus, and Alternaria tenuis in the autumn group $(P<0.001)$.

Conclusions These results suggest that house dust is the main cause of allergic conjunctivitis during autumn. In spring, cypress pollen is the largest cause of allergic conjunctivitis, while indoor allergens such as house dust, animal epithelia, D. pteronyssinus, and acarus are not causative allergens in Japan.

Eye (2005) 19, 995-999. doi:10.1038/sj.eye.6701701; published online 24 September 2004
\end{abstract}

Keywords: allergic conjunctivitis; CAP-RAST system; house dust; specific IgE; total IgE

\section{Introduction}

Seasonal allergy in itself is a common disease. Various allergens can cause allergic diseases
T Mimura', 2, S Yamagami', S Amano', H Funatsu3 ${ }^{3}$, A Arimoto ${ }^{1,2}$, T Usui $^{1}, \mathrm{~K}^{1} \mathrm{Ono}^{1}$, M Araie ${ }^{1}$ and S Okamoto ${ }^{2}$

and the clinical symptoms of the allergic state can appear in the forms of conjunctivitis, rhinitis, or bronchial asthma. ${ }^{1-3}$ Allergic conjunctivitis is the commonest manifestation of sensitization. As so many people suffer from allergic conjunctivitis, it is crucial to detect the causal allergens. The annual incidence of allergic conjunctivitis peaks twice, once in spring and again in autumn in Japan. Cedar and cypress pollen have been identified as the major allergen sources of allergic conjunctivitis in spring. ${ }^{4}$ However, little is known about causative allergens of allergic conjunctivitis in autumn.

The aim of the current study is to evaluate the relation between specific immunoglobulin $\mathrm{E}$ (IgE) in serum and allergic conjunctivitis in autumn using the CAP radioallergosorbent test (CAP-RAST) system. Additionally, we investigated the correlation between total and specific IgE in patients with allergic conjunctivitis raised against various allergens in autumn.

\section{Materials and methods}

\section{Subject}

This study was performed in accordance with the Helsinki Declaration of 1975 and its 1983 revision. All participants provided written informed consent before being tested.

Outpatients with allergic conjunctivitis, who had been treated at Okamoto Eye Clinic, were selected for this trial. The CAP-RAST system (Phadiatop $^{\mathrm{TM}}$, Pharmacia, Uppsala, Sweden) and the determination of total $\operatorname{IgE}$ levels were performed in duplicate on sera obtained from each of the subjects in the two groups. Group One, the spring group, consisted of 32 patients suffering from acute seasonal allergic conjunctivitis just in spring $(10 \mathrm{M}, 22 \mathrm{~F}$, mean age $52.8 \pm 12.5$ years, range $16-68$ years) who
${ }^{1}$ Department of Ophthalmology University of Tokyo Graduate School of Medicine

Tokyo, Japan

${ }^{2}$ Okamoto Eye Clinic Yamato, Japan

${ }^{3}$ Department of Ophthalmology Diabetes Center Tokyo Women's Medical University Tokyo, Japan

Correspondence: T Mimura 7-3-1 Hongo, Bunkyo-ku Tokyo 113-8655, Japan Tel: + 81358005108 ext 33503

Fax: +81338170798

E-mail:mimura@

mbg.ocn.ne.jp

Received: 1 March 2004 Accepted: 13 July 2004 Published online: 24 September 2004

The authors have no commercial or proprietary interest in the product or company described in the current article. 
were treated only between February and April 2002. Group Two, the autumn group, consisted of 27 patients suffering allergic conjunctivitis just in autumn $(9 \mathrm{M}, 18 \mathrm{~F}$ mean age $49.3 \pm 16.7$ years, range $13-71$ years) who were treated only between August and October 2002. Group Three, the control group, consisted of 40 healthy, nonsmoker volunteers with no history of allergic diseases. All had negative skin-prick test results (Phazet ${ }^{\circledR}$, Pharmacia), none wore contact lenses or had been treated with a topical or systemic drug, in the preceding 6 weeks (17 M, $23 \mathrm{~F}$, mean age, $40.2 \pm 11.1$ years, range $20-58$ years). Allergic conjunctivitis was diagnosed according to the guidelines of diagnosis and treatment of conjunctivitis. ${ }^{5}$ We excluded patients with atopic conjunctivitis and infectious conjunctivitis and patients who had atopic disorders such as asthma or allergic rhinitis from this trial. IgE levels were compared among these three groups.

\section{Allergens}

In all, 12 RAST were performed using the following allergens: a mixture in equal parts of five-animal epithelia (cat epithelium/dander, dog, guinea-pig, rat, mouse), house dust, Dermatophagoides pteronyssinus, acarus, moth, Candida, Alternaria tenuis, Japanese cedar (Cryptomeria japonica) pollen, Japanese cypress (Chamaecyparis obtuse) pollen, ragweed, mugwort, and orchard grass. These groups include the allergens that are the main causes of allergy in Japan.

\section{Determination of total and specific IgE in serum}

The determination of total IgE was performed with PRIST (Phadezym IgE PRIST ${ }^{\circledR}$, Pharmacia). Blood was drawn for an assay on specific IgEs in the serum using the Pharmacia CAP System. Assays were carried out on undiluted sera against each of the 12 allergens. The assays were carried out in duplicate and the radioimmunoassay version of the Pharmacia CAP System used. ${ }^{6-8}$ The manufacturer's instructions were strictly followed. In brief, test sera and standards were incubated at room temperature (approximately $20^{\circ} \mathrm{C}$ ) with the ImmunoCAP for $30 \mathrm{~min}$, then washed. IgE (Fc)-specific combinations of polyclonal and monoclonal anti-IgE labelled with ${ }^{125}$ I were added. After a second incubation step of $150 \mathrm{~min}$, the ImmunoCAP was washed, and the bound radioactivity counted in a gamma counter. The specific IgE assay was calibrated against a WHO standard for IgE, in the range $0.35-100 \mathrm{kU} / 1$. Results were read from a standard curve. The upper and lower cutoff points were 100 and $0.35 \mathrm{kU} / 1$ as for the Phadebas RAST, and the results could be expressed either as $\mathrm{kU} / \mathrm{l}$ or as classes $(0-6$, as for RAST but where class 0 is $<0.35 \mathrm{kU} / 1$, class 1 is equivalent to $0.35-0.69 \mathrm{kU} / \mathrm{l}$, class 2 is to $0.70-3.49 \mathrm{kU} / 1$, class 3 is to $3.50-17.49 \mathrm{kU} / 1$, class 4 is to $17.50-49.99 \mathrm{kU} / \mathrm{l}$, class 5 is equivalent to $50.00-100.00 \mathrm{kU} / \mathrm{l}$, and class 6 is $>100 \mathrm{kU} / \mathrm{l}$ ). The solid-phase allergens in the ImmunoCAP were optimized to give maximum binding with low background levels, even when undiluted test sera were used.

\section{Statistical analysis}

The unpaired $t$-test was performed to compare mean values between the two groups. The one-way analysis of variance and Scheffe's multiple comparison were used to compare the mean values among the three groups. The level of significance was set at $P<0.05$. The relation between continuous variables was investigated by means of Pearson's correlation coefficient and partial correlation coefficient. The correlation coefficient was evaluated by Fisher's $Z$ transformation and the Bartlett test. As for the total and specific IgE, all analyses should be based on log-transformed data. Calculations were made using the Stat View statistical software package (Abacus Concepts, Berkeley, CA, USA).

\section{Results}

Total IgE was higher in the autumn group than in the spring group and the control group (Table 1). Specific IgE antibody levels against house dust, D. pteronyssinus, acarus, moth and $A$. tenuis were higher in the autumn group than in the spring group and the control group (Table 1). There was no difference in all specific IgE antibody levels and total IgE levels between male and female patients.

The positive frequencies of specific IgE antibodies against 12 allergens were determined by CAP-RAST (Figure 1). The highest positive rate was $51.9 \%$ for house dust, followed by D. pteronyssinus $(48.1 \%)$ and cedar pollen $(44.4 \%)$ in the autumn group. In contrast, the positive rates for Candida, Alternaria, and animal epithelia were low. In the spring group, the highest rate was cedar pollen $(68.8 \%)$, followed by cypress pollen $(59.4 \%)$.

Specific IgE antibody levels were compared between the 14 house dust-positive and 13 house dust-negative patients. The levels of total IgE and all the specific IgEs showed a highly significant difference between the positive and negative groups (Table 2).

Correlation analysis was performed between each specific IgE antibody level in the autumn group (Table 3). Using both Pearson's correlation coefficient and partial correlation coefficient, house dust was significantly correlated with animal epithelia, D. pteronyssinus, acarus, and $A$. tenuis $(P<0.0001)$. On the other hand, cedar 
Table 1 Comparisons of various specific IgE among the control group, the spring group and the autumn group

\begin{tabular}{|c|c|c|c|c|}
\hline & Control & Spring & Autumn & \\
\hline Number of patients & 40 & 32 & 27 & \\
\hline Age (years) & $40.2 \pm 11.1$ & $52.8 \pm 12.5$ & $49.3 \pm 16.7$ & \\
\hline \multirow[t]{2}{*}{ Male/female } & $17 \overline{23}$ & $10 / \overline{2} 2$ & $9 / \overline{18}$ & \\
\hline & $\log (\operatorname{IgE}$ level $)$ & $\log (\operatorname{IgE}$ level $)$ & $\log (\operatorname{IgE}$ level $)$ & $\mathrm{P}$ \\
\hline Total IgE & $0.92 \pm 0.32$ & $1.69 \pm 0.61$ & $1.90 \pm 0.68$ & $<0.001$ \\
\hline Animal epithelia & $-1.06 \pm 0.34$ & $-0.99 \pm 0.45$ & $-1.01 \pm 0.58$ & NS \\
\hline House dust & $-1.09 \pm 0.33$ & $-0.77 \pm 0.55$ & $-0.42 \pm 0.83$ & $<0.001$ \\
\hline D. pteronyssinus & $-0.93 \pm 0.37$ & $-0.63 \pm 0.41$ & $-0.36 \pm 0.77$ & $<0.001$ \\
\hline Acarus & $-1.09 \pm 0.38$ & $-0.79 \pm 0.58$ & $-0.58 \pm 0.81$ & 0.003 \\
\hline Moth & $-1.02 \pm 0.30$ & $-0.64 \pm 0.51$ & $-0.55 \pm 0.65$ & $<0.001$ \\
\hline Candida & $-0.98 \pm 0.27$ & $-0.86 \pm 0.37$ & $-0.91 \pm 0.46$ & NS \\
\hline A. tenuis & $-1.03 \pm 0.23$ & $-0.86 \pm 0.29$ & $-0.82 \pm 0.35$ & 0.007 \\
\hline Cedar pollen & $-0.80 \pm 0.41$ & $0.25 \pm 0.99$ & $-0.25 \pm 0.99$ & $<0.001$ \\
\hline Cypress pollen & $-0.98 \pm 0.41$ & $-0.39 \pm 0.57$ & $-0.81 \pm 0.77$ & $<0.001$ \\
\hline Ragweed & $-0.85 \pm 0.43$ & $-0.67 \pm 0.52$ & $-0.59 \pm 0.78$ & NS \\
\hline Mugwort & $-0.91 \pm 0.21$ & $-0.90 \pm 0.51$ & $-0.75 \pm 0.69$ & NS \\
\hline Orchard grass & $-1.07 \pm 0.39$ & $-0.95 \pm 0.41$ & $-0.87 \pm 0.63$ & NS \\
\hline
\end{tabular}

Number of patients or mean \pm standard deviation. NS, not significant.
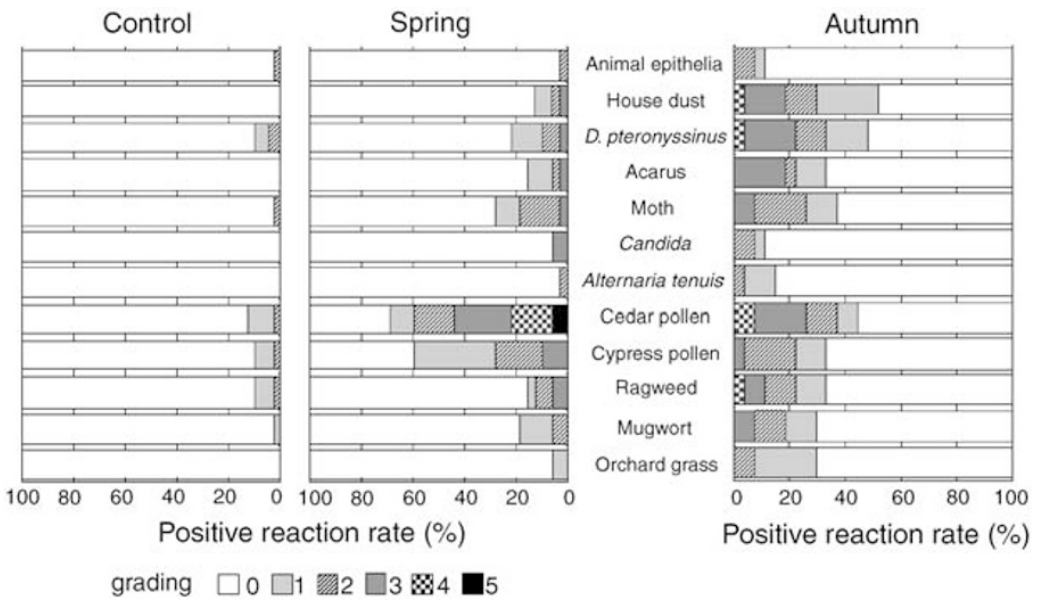

Figure 1 Frequency of IgE antibodies and grading of IgE. Frequency of IgE antibodies against 12 allergens determined by CAP-RAST in the spring group $(n=32)$ and the autumn group $(n=27)$.

pollen was correlated with cypress, ragweed, mugwort, and orchard grass $(P<0.0001)$.

It was confirmed by the Bartlett test that there was a correlation between some variables in the autumn group $\left(\chi^{2}=420.1, P<0.0001\right)$.

\section{Discussion}

The specific IgE levels and the positive frequency of specific IgE against house dust, D. pteronyssinus, acarus, moth, cedar pollen, cypress pollen, and ragweed were found to be high in the autumn group, suggesting that these allergens are causes of allergic conjunctivitis in autumn. There are two continuous cedar pollen seasons with high pollen levels in Japan. Fujishima et $a l^{9}$ reported that cedar pollen is scattered not only in spring but in all seasons, especially in the autumn in Japan, consequently cedar pollen can cause allergic conjunctivitis both in the spring and late autumn. In addition, there is the mugwort and ragweed pollen season from August until October, but the pollen levels are much lower than in cedar and cypress pollen. The symptoms of seasonal allergic conjunctivitis that develop from spring to summer are generally caused by cedar and cypress pollen. In contrast, the year-round allergens also cause allergic conjunctivitis from spring to early autumn. This 
Table 2 Comparison of $\operatorname{IgE} /$ specific IgE between house dust-positive and -negative allergic conjunctivitis patients in autumn

\begin{tabular}{|c|c|c|c|c|c|c|c|}
\hline & \multicolumn{3}{|c|}{ House dust (+) $\mathrm{N}=14$} & \multicolumn{3}{|c|}{ House dust (-) $\mathrm{N}=13$} & \multirow[t]{2}{*}{$\mathrm{t}$-Test } \\
\hline & $\log (\operatorname{IgE}$ level $)$ & $\mathrm{N}$ & $(\%)$ & $\log (\operatorname{IgE}$ level $)$ & $\mathrm{N}$ & $(\%)$ & \\
\hline Total IgE & $2.34 \pm 0.47$ & & & $1.42 \pm 0.51$ & & & $<0.001$ \\
\hline Animal epithelia & $-0.74 \pm 0.51$ & 3 & $(21.4 \%)$ & $-1.13 \pm 0.31$ & 0 & $(0.0 \%)$ & 0.015 \\
\hline House dust & $0.19 \pm 0.55$ & 14 & $(100.0 \%)$ & $-0.97 \pm 0.32$ & 0 & $(0.0 \%)$ & $<0.001$ \\
\hline D. pteronyssinus & $0.21 \pm 0.56$ & 13 & $(92.9 \%)$ & $-0.97 \pm 0.33$ & 0 & $(0.0 \%)$ & $<0.001$ \\
\hline Acarus & $-0.04 \pm 0.64$ & 9 & $(64.3 \%)$ & $-1.12 \pm 0.40$ & 0 & $(0.0 \%)$ & $<0.001$ \\
\hline Moth & $-0.30 \pm 0.56$ & 7 & $(50.0 \%)$ & $-0.76 \pm 0.51$ & 3 & $(23.1 \%)$ & 0.022 \\
\hline Candida & $-0.72 \pm 0.36$ & 3 & $(21.4 \%)$ & $-1.02 \pm 0.28$ & 0 & $(0.0 \%)$ & 0.013 \\
\hline A. tenuis & $-0.68 \pm 0.31$ & 3 & $(21.4 \%)$ & $-0.96 \pm 0.32$ & 1 & $(7.7 \%)$ & 0.018 \\
\hline Cedar pollen & $0.40 \pm 0.80$ & 10 & $(71.4 \%)$ & $-0.90 \pm 0.50$ & 2 & $(15.4 \%)$ & $<0.001$ \\
\hline Cypress pollen & $-0.45 \pm 0.65$ & 8 & $(57.1 \%)$ & $-1.01 \pm 0.52$ & 1 & $(7.7 \%)$ & 0.013 \\
\hline Ragweed & $-0.28 \pm 0.72$ & 6 & $(42.9 \%)$ & $-0.88 \pm 0.63$ & 3 & $(23.1 \%)$ & 0.018 \\
\hline Mugwort & $-0.35 \pm 0.51$ & 7 & $(50.0 \%)$ & $-1.01 \pm 0.35$ & 1 & $(7.7 \%)$ & $<0.001$ \\
\hline Orchard grass & $-0.61 \pm 0.43$ & 5 & $(35.7 \%)$ & $-0.99 \pm 0.52$ & 1 & $(7.7 \%)$ & 0.029 \\
\hline
\end{tabular}

$\log (\mathrm{IgE}$ level) (mean \pm standard deviation) or number $(N)$ of IgE-positive patients. Numbers in parentheses are percentages. NS, not significant.

Table 3 Correlation coefficients between allergens in allergic conjunctivitis patients in autumn

\begin{tabular}{|c|c|c|c|c|c|c|c|c|c|c|c|c|}
\hline & $\operatorname{IgE}$ & & & & & & & & & & & \\
\hline Epithelia & 0.122 & Epithelia & & & & & & & & & & \\
\hline House dust & ${ }^{\dagger} 0.623$ & ${ }^{\dagger} 0.594$ & House dust & & & & & & & & & \\
\hline Acarus & ${ }^{\dagger} 0.607$ & $* * 0.533$ & ${ }^{\dagger} 0.812$ & ${ }^{\dagger} 0.820$ & Acarus & & & & & & & \\
\hline Moth & $* 0.337$ & $\dagger^{\dagger} 0.589$ & $* * 0.472$ & ${ }^{*} 0.440$ & $* * 0.465$ & Moth & & & & & & \\
\hline Candida & 0.123 & ${ }^{\dagger} 0.840$ & ${ }^{* *} 0.463$ & ${ }^{* *} 0.464$ & ${ }^{*} 0.427$ & ${ }^{\dagger} 0.720$ & Candida & & & & & \\
\hline Alternaria & ${ }^{*} 0.327$ & $* * 0.562$ & †0.586 & ${ }^{\dagger} 0.612$ & ${ }^{\dagger} 0.592$ & $* 0.414$ & ${ }^{\dagger} 0.571$ & Alternaria & & & & \\
\hline Cedar & ${ }^{* *} 0.489$ & ${ }^{*} 0.438$ & ${ }^{* *} 0.527$ & $* * 0.543$ & $* * 0.558$ & ${ }^{*} 0.425$ & ${ }^{* *} 0.548$ & ${ }^{* *} 0.460$ & Cedar & & & \\
\hline Cypress & ${ }^{*} 0.354$ & ${ }^{*} 0.401$ & 0.285 & 0.267 & 0.293 & $* * 0.545$ & $* * 0.530$ & 0.284 & ${ }^{\dagger} 0.821$ & Cypress & & \\
\hline Ragweed & $* 0.372$ & $* 0.393$ & *0.398 & ${ }^{*} 0.406$ & $* * 0.473$ & $* * 0.529$ & $* * 0.501$ & $* * 0.513$ & 0.630 & 0.612 & Ragweed & \\
\hline Mugwort & $* 0.410$ & $* * 0.546$ & ${ }^{* *} 0.481$ & ${ }^{* *} 0.489$ & $* * 0.487$ & ${ }^{\dagger} 0.636$ & ${ }^{\dagger} 0.658$ & $* * 0.560$ & ${ }^{\dagger} 0.774$ & 0.737 & †0.803 & Mugwort \\
\hline Orchard grass & 0.222 & ${ }^{\dagger} 0.723$ & ${ }^{*} 0.397$ & ${ }^{*} 0.383$ & $* 0.369$ & ${ }^{\dagger} 0.578$ & ${ }^{\dagger} 0.776$ & ${ }^{* *} 0.484$ & ${ }^{\dagger} 0.650$ & ${ }^{\dagger} 0.693$ & 0.725 & ${ }^{\dagger} 0.806$ \\
\hline
\end{tabular}

Correlation coefficients were calculated by Pearson's product moment formula.

${ }^{*} P<0.05,{ }^{* *} P<0.01,{ }^{\dagger} P<0.001$.

is not only because it is the pollen season of various plants such as ragweed, mugwort, and orchard grass, but also because mites proliferate in the high temperatures and high humidity of this season. Patients who were positive for house dust have a high IgE level for other antigens. Therefore, there is multiple antigen sensitivity in this group.

There are many kinds of specific IgEs that correlate with each other. House dust tends to be associated with animals, mites, insects, and moulds such as animal epithelia, D. pteronyssinus, acarus, moth, candida, and A. tenuis. In contrast, allergy to cedar pollen appeared to be associated with the sensitivity to pollens such as cypress, ragweed, and mugwort. Allergens can be divided into two categories according to their indoor or outdoor source. House dust mites are the major source of indoor allergens followed by animal danders. Cedar and cypress pollen are typical outdoor allergens follows by moth. Indoor allergens may be more frequently associated with perennial allergic conjunctivitis, while outdoor ones are more closely related to seasonal allergic conjunctivitis. These clinically significant associations indicate that crossreacting allergens of different kinds are present in these allergens, suggesting the presence of at least some epitopes to both allergens. A significant correlation was found between the specific IgE levels of house dust and D. pteronyssinus $(P<0.0001)$ and house dust and acarus $(P<0.0001)$ by both Pearson's and partial correlation coefficient. D. pteronyssinus and acarus are sarcoptes, which cause a long pathologic antigenantibody reaction in the human body. The high correlations between house dust and D. pteronyssinus and between house dust and acarus indicate that mites occupy an important position in house dust. The elimination of house dust will therefore lead to elimination of the mite. 
Our results confirm that house dust mite-specific IgE production is closely associated with conjunctival allergy in autumn. These findings also have clinical significance, consequently we can employ the correlation results shown in this study to advise patients on which allergens to avoid and treat in advance in the season with high positive antigen levels.

\section{References}

1 Suzuki M, Itoh H, Sugiyama K, Takagi I, Nishimura J, Kato K et al. Causative allergens of allergic rhinitis in Japan with special reference to silkworm moth allergen. Allergy 1995; 50: 23-27.

2 Plebani M, Faggian D, Borghesan F. Full automation in allergy testing: measurement of specific IgE by the ENEA System. Allergy 1995; 50: 229-233.

3 Kerkhof M, Schouten JP, de Monchy JG. The association of sensitization to inhalant allergens with allergy symptoms: the influence of bronchial hyperresponsiveness and blood eosinophil count. Clin Exp Allergy 2000; 30: 1387-1394.

4 Mimura T, Amano S, Funatsu H, Yamagami S, Araie M, Kaji Y et al. Correlations between allergen-specific IgE serum levels in patients with allergic conjunctivitis in spring. Ocul Immunol Inflam 2004; 12: 45-51.

5 Ben Ezra D. Guidelines on the diagnosis and treatment of conjunctivitis. Ocul Immunol Inflam 1994; 2(Suppl): $17-26$.

6 Axen R, Drevin H, Kober A, Yman L. A new laboratory diagnostic system applied to allergy testing (abstract). Allergy Proc 1998; 9: 503.

7 Duc J, Peitrequin R, Pcoud A. Value of a new screening test for respiratory allergy. Allergy 1988; 43: 332-337.

8 Ewan PW, Coote D. Evaluation of a capsulated hydrophilic carrier polymer (the ImmunoCAP) for measurement of specific IgE antibodies. Allergy 1990; 45: 22-29.

9 Fujishima H, Sahashi N, Shimazaki J, Tsubota K. Allergic conjunctivitis caused by sugi (Cryptomeria japonica D. Don) pollen out of season. Asian Pac J Allergy Immunol 1995; 13: 113-117. 